(EL-LRTI+ve versus EL-LRTI-ve) was greater in non-smokers $(\mathrm{OR}=4.0(95 \%$ confidence interval $(\mathrm{CI}): 1.9$ to $8.2 ; \mathrm{P}<0.001)$ than in smokers $(\mathrm{OR}=1.48 \mathrm{CI}$ : 0.8 to $2.6 ; \mathrm{P}=0.17)$. In nonsmokers only the association between EL-LRTI and adult CMH lessened with ageing. Thus by age 60, the effect of EL-LRTI on the odds of having CMH (EL-LRTI+ve versus EL-LRTI-ve) amongst non-smokers $(\mathrm{OR}=1.4(\mathrm{CI}: 0.8$ to $2.4 ; \mathrm{P}=0.21))$ and smokers $(\mathrm{OR}=1.6 \mathrm{CI}: 0.8$ to $3.1 ; \mathrm{P}=0.15)$ was similar.

Conclusion Infants with respiratory infections become adults predisposed to developing $\mathrm{CMH}$ potentially reflecting pulmonary damage sustained during early-life or an overarching altered susceptibility to respiratory insults. This relationship is modulated by smoking and ageing.

\section{T3 HUMAN RHINOVIRUS IMPAIRS THE INNATE IMMUNE RESPONSE TO BACTERIA IN MONOCYTE DERIVED MACROPHAGES FROM PATIENTS WITH CHRONIC OBSTRUCTIVE PULMONARY DISEASE}

U Finney, KBR Belchamber, P Mallia, SL Johnston, LE Donnelly, JA Wedzicha. National Heart and Lung Institute, Imperial College, London, UK

\subsection{6/thoraxjnl-2016-209333.3}

Introduction Exacerbations of Chronic Obstructive Pulmonary Disease (COPD) are associated with accelerated disease progression, hospitalisation and death. Respiratory viruses are identified in approximately half of all exacerbations. We have previously found that human rhinovirus infection leads to a secondary outgrowth of bacteria which is associated increased exacerbation severity. The mechanisms of how HRV increases risk of secondary bacterial outgrowth are unknown.

Hypothesis We hypothesised that HRV infection impairs phagocytosis of bacteria by monocyte derived macrophages (MDM) which may lead to an increased risk of secondary bacterial outgrowth during COPD exacerbations.

Methods Participants were recruited from the London COPD Cohort. MDM were generated by culture in GM-CSF or M-CSF for 12 days. MDM were incubated with HRV 16 for 24 hours at increasing multiplicity of infection (MOI) $0.5-10$ for 24 hours or poly-IC at increasing concentrations $0-300 \mu \mathrm{g} / \mathrm{ml}$.

Phagocytic capacity was then assessed by incubating MDMs with fluorescently labelled heat killed Haemophilus influenzae or Streptococcus pneumoniae for 4 hours and uptake measured by fluorimetry.

The pro-inflammatory cytokine CXCL- 8 and anti-inflammatory cytokine IL-10 were measured by ELISA according to the manufacturer's instructions.

Results HRV16 impaired phagocytosis of H. influenzae (HRV (MOI of 5) $2.84 \pm 0.92$ vs media control $4.36 \pm 1.06 \mathrm{RFU} \times$ $\left.10^{3} \mathrm{n}=8, \mathrm{p}=0.01\right)$ and $S$. pneumoniae $(\mathrm{p}<0.01)$ by MDM in a virus-dose- dependent manner without impairing cell viability. HRV16 alone induced CXCL-8 and IL-10 release from MDM compared to media alone. HRV16 (MOI 5) significantly impaired IL-10 response to $H$. influenzae compared to media alone $(0.59$ $(0.33-0.96) \mathrm{ng} / \mathrm{ml}$ vs $1.83(1.11-3.00) \mathrm{ng} / \mathrm{ml}$ respectively, $\mathrm{n}=6$, $\mathrm{p}=0.03)$ and impaired CXCL-8 response to $H$ influenzae compared to media alone $4.41(3.45-5.85) \mathrm{ng} / \mathrm{ml}$ vs 24.65 (11.6329.77) $\mathrm{ng} / \mathrm{ml}$ respectively, $\mathrm{n}=5, \mathrm{p}=0.01)$.
Poly-IC impaired phagocytosis of $H$. influenzae in a concentration-dependent manner without significantly impairing cell viability. Poly IC alone also induced IL-8 release from MDM.

Conclusions HRV impairs phagocytosis of bacteria by MDM in COPD and impairs cytokine response to bacteria which may inhibit neutrophil influx and prevent resolution of inflammation. This may lead to an outgrowth of bacteria and prolonged exacerbations in COPD.

\section{T4 GLOBAL SPREAD OF MYCOBACTERIUM ABSCESSUS CLONES AMONGST CYSTIC FIBROSIS PATIENT}

${ }^{1} \mathrm{DM}$ Grogono, ${ }^{2} \mathrm{JM}$ Bryant, ${ }^{1} \mathrm{D}$ Rodriguez-Rincon, ${ }^{2}$ Everall, ${ }^{3} \mathrm{KP}$ Brown, ${ }^{4} \mathrm{P}$ Moreno, ${ }^{5} \mathrm{D}$ Verma, ${ }^{5} \mathrm{E}$ Hill, ${ }^{1}$ J Drijkoningen, ${ }^{3} \mathrm{CS}$ Haworth, ${ }^{2} \mathrm{SR}$ Harris, ${ }^{5} \mathrm{D}$ Ordway, ${ }^{2} \mathrm{~J}$ Parkhill, ${ }^{1}$ RA Floto. ${ }^{1}$ University of Cambridge Department of Medicine, Cambridge, UK; ${ }^{2}$ Wellcome Trust Sanger Institute, Hinxton, UK; ${ }^{3}$ Cambridge Centre for Lung Infection, Papworth Hospital, Papworth, UK; ${ }^{4}$ EMBL European Bioinformatics Institute, Hinxton, UK; ${ }^{5}$ Mycobacteria Research Laboratory, Department of Microbiology, Immunology and Pathology, Colorado State University, Fort Collins, USA

\subsection{6/thoraxjnl-2016-209333.4}

Introduction Lung infections with Mycobacterium abscessus, a species of multidrug resistant nontuberculous mycobacteria, have increased in frequency worldwide, emerging as an important global threat to individuals with cystic fibrosis (CF) where they cause accelerated inflammatory lung damage and death. $M$. abscessus was previously thought to be independently acquired by susceptible individuals from the environment. However, using whole genome sequencing and detailed epidemiological analysis of a cohort of patients attending the CF centre at Papworth Hospital, we found strong evidence for transmission between patients. We therefore sought to examine the mechanism of acquisition of M. abscessus in CF individuals across the world.

Methods We undertook whole genome sequencing on 1080 isolates from 517 patients from the UK, US, the Republic of Ireland, mainland Europe and Australia. This was then correlated with clinical metadata and phenotypic functional analysis.

Results Our genomic analysis revealed that the majority of infections are from densely clustered M. abscessus genotypes with low levels of diversity, indicating a high level of human associated spread. Moreover, the phylogeny reveals the presence of three recently emerged dominant circulating clones that have globally spread. We found that these clones are associated with worse clinical outcomes and show increased virulence in both cell-based and mouse infection models. Within patients we found evidence of genetic diversity and evolutionary adaptation through the processes of convergent evolution and hypermutation.

Conclusions The majority of $M$. abscessus infections in patients with Cystic Fibrosis are caused by genetically related clusters, indicating recent patient-to-patient transmission despite conventional infection control measures. Transmission appears to have facilitated evolution of $M$. abscessus from an environmental organism into a transmissible human pathogen.

This work was supported by The Wellcome Trust grant 098051 (JMB, SH, JP) and 107032AIA (DG, RAF) The Medical Research Council (JMB), The UK Cystic Fibrosis Trust (DMG, DR-R, IE, JP, RAF), Papworth Hospital (DMG, KPB, CSH, RAF), NIHR Cambridge Biomedical Research Centre (RAF), and The UKCRC Translational Infection Research Initiative (JP). 